\title{
Traduire les énoncés religieux indigénisés: cas de la traduction du cilubà en français et en anglais*
}

\author{
Germain Mulamba Nshindi, Institut Supérieur Pédagogique, Mbujimayi, \\ République Démocratique du Congo (ngmulamba@hotmail.com / \\ sgacispmjm@yahoo.fr)
}

\begin{abstract}
Résumé: Cet article s'efforce à examiner les difficultés inhérentes à la traduction du cilubà en français et en anglais de certains énoncés, à savoir, les expressions religieuses, les devises et les proverbes. Il a essayé de démontrer qu'une fois que le message biblique a été indigénisé en cilubà, il devient très difficile de le traduire à nouveau dans les langues européennes, en l'occurrence en français et en anglais. En effet, il contient des «africanismes» ou des réalités locales qui sont puisées dans la cosmologie africaine, laquelle n'est pas toujours conforme à la foi chrétienne. Enfin, l'emploi de l'ethnographie de communication du cilubà pendant les cérémonies religieuses incite à l'emploi des métaphores, des proverbes et autres aspects du langage imagé. À moins de recourir à la traduction fonctionnelle, l'interprète ne peut pas aider les locuteurs français et anglais à participer à ces pratiques langagières.
\end{abstract}

Mots clés: ÉNONCÉ, DEVISE, CONTEXTE, INDIGÉNISATION, RENOUVEAU LITURGIQUE, TRADUCTION, TRADUCTOLOGIE, PARÉMIES, ETHNOGRAPHIE DE COMMUNICATION, LANGAGE IMAGÉ

\begin{abstract}
Translating Indigenized Religious Utterances: A Case of the Translation of Cilubà into French and English. This article is an attempt at examining the difficulties inherent in translating certain utterances, namely religious expressions, praise names and proverbs, from Cilubà into French and English. It tries to show that once the Biblical message has been indigenized in Cilubà, it becomes very difficult to translate it back again into the European languages, in this case French and English. In fact, it contains "Africanisms" or local realities which draw on African cosmology which is not always in conformity with the Christian faith. Finally, the use of the ethnography of communication of Cilubà during religious ceremonies leads people to turn to metaphors, proverbs and other aspects of figurative language. Unless turning to functional translation, the interpreter cannot assist French and English speakers to participate in these speech events.
\end{abstract}

Keywords: UTTERANCE, PRAISE NAME, CONTEXT, INDIGENIZATION, LITURGICAL REVIVAL, TRANSLATION, TRANSLATION THEORY, PROVERBS, ETHNOGRAPHY OF COMMUNICATION, FIGURATIVE LANGUAGE

* Cet article est une version adaptée de notre communication «Le traducteur face au renouveau liturgique» présentée au Colloque Théologique organisé par la Faculté de Théologie Protestante de Mbujimayi, République Démocratique du Congo, 3-5 janvier 2000. 


\section{Introduction}

Cet article essaie d'examiner les difficultés inhérentes à la traduction du cilubà en français et en anglais des énoncés religieux indigénisés couramment utilisés dans les cantiques, les discours et les textes de la liturgie catholique romaine. Le cilubà est une langue bantu parlée dans les deux provinces du Kasaï en République Démocratique du Congo. Il est connu sous le sigle linguistique L31, selon la classification de Guthrie (1948).

En effet, parmi les innovations remarquables de ces dernières décennies dans l'Église Catholique Romaine d'Afrique figure «le renouveau liturgique». Il se caractérise entre autres par l'africanisation du rite religieux, l'inculturation du message biblique et linguistiquement, par l'utilisation des langues locales en remplacement du latin et des autres langues européennes. À la suite des sociolinguistes, nous pouvons parler de «l'indigénisation» (Kachru 1982) de la Bonne Nouvelle. Selon Kabasélé (1986a: 270), «inculturer la foi signifie insérer le message chrétien dans une culture, y adhérer avec ses modes de penser, d'agir, de vivre; avec ce qu'on est et aspire à être ...»

$\mathrm{Au}$ cœur de cette entreprise se trouvent deux éminences africaines, à savoir le Cardinal Albert Joseph Malula de la République Démocratique du Congo et le Révérend Père Englebert Mveng du Cameroun.

Bien plus, sur le plan de la praxis langagière, ces langues sont utilisées conformément à l'ethnographie de communication spécifique à chaque langue et à chaque culture africaines. En d'autres termes, cette pratique langagière va au-delà de la simple traduction du message biblique européen et devient ancrée dans la créativité africaine pour atteindre le fond de la pensée et de la croyance des africains.

À ce moment où l'interprétation directe ou traduction simultanée est devenue une pratique courante dans la plupart des confessions religieuses, nous avons jugé nécessaire de cogiter sur la traduction du cilubà en français et en anglais de certains énoncés utilisés lors du rite religieux catholique. Notre objectif est d'apprécier le degré d'inculturation de la Bonne Nouvelle dans cette culture africaine et les difficultés potentielles de sa réexpression de ce message indigénisé en français et en anglais.

En effet, sur le plan lexical et discursif, le continuum du registre religieux catholique lubà englobe des mots d'emprunt d'origine latine, des mots altérés ou assimilés en cilubà, et des lexèmes purement natifs n'ayant aucun lien apparent avec les textes européens. Kabasélé fait le même constat dans sa classification des «devises» concernant Jésus, c'est-à-dire, «des syntagmes épithétiques élogieux, plus ou moins figés, qui ... accompagnent ou remplacent le nom du héros» (Faiik-Nzuji 1974: 14). Ainsi identifie-t-il «les titres qui sont puisés dans (ou inspirés de) la Révélation ..., ceux empruntés à la Tradition Bantu et enfin ceux issus soit d'une synthèse entre la Tradition Bantu et la Bible, soit d'une simple coïncidence ou juxtaposition des deux» (Kabasélé 1986a: 264) comme illustré ci-dessous: 
(a) Devises tirés de la Révélation:

Mulami mulenga: Le Bon Pasteur (The Good Shepherd)

Ntambwa wa cisàmbà cyà Yuudà: Le Lion de la tribu de Judée (The Lion of the tribe of Judaea)

(b) Devises tirés de la Tradition bantu:

Nsànga mweyèmèna Mulopò: Le chêne appuyé sur Dieu (The oak supported by God)

Ngabu wa ncibula bilòbò: Le bouclier sur qui se brisent les héros (The shield on which the brave warriors are broken)

(c) Devises tirés de la Tradition bantu et la Bible:

Mufwà mwalùka wa Maweja: La mort revenu de Dieu (The Resurrection of God)

Mfùmù, Mukalenga, Mulopò: Chef, Seigneur (Chief, Lord)

Ce continuum révèle aussi les divers degrés de la profondeur d'inculturation des lexèmes religieux en cilubà. Les lexèmes natifs font partie du domaine du langage imagé, langage difficile à traduire en langues européennes à cause de différences culturelles très prononcées. Notre but est de démontrer qu'une fois inculturé, le texte liturgique en cilubà devient difficile à restituer en langues européennes, en français et en anglais pour le cas d'espèce. Le traducteur a besoin d'une bonne connaissance de la cosmogonie lubà et d'une bonne maîtrise des stratégies de traduction. Certes, une telle entreprise nécessite non plus la traduction presque littérale typique à la Bible, c'est-à-dire «la traduction sémantique», mais souvent la «traduction fonctionnelle» ou traduction culturelle (Newmark 1982).

Nous arguons qu'en acceptant l'utilisation des langues africaines dans les rites religieux, on a aussi libéré le langage du croyant, et partant, le croyant luimême. D'où l'emploi du langage imagé et de l'ethnographie de communication propres à la culture des croyants.

À cette fin, nous esquisserons d'abord la théorie de la traduction et l'ethnographie de communication. Ensuite, nous procéderons à la traduction en français et en anglais des quelques énoncés lubà. Il s'agit principalement des expressions, des devises et des métaphores. Par ailleurs, nous traduirons aussi en français et en anglais quelques expressions lubà relatives à l'ethnographie de communication pour illustrer l'indigénisation du rite religieux par la pratique langagière. La conclusion résumera l'essentiel de notre argument.

\section{Considérations méthodologiques}

Cette étude focalise à la fois sur la théorie de traduction et sur l'ethnographie de communication. Elle est basée sur des données écrites et orales, à savoir les textes liturgiques, les prières, les cantiques, les prêches et les commentaires des modérateurs des messes. Ainsi, tenterons-nous de traduire un échantillon d'é- 
noncés courants dans ces données. Cependant, contrairement aux études traductologiques qui examinent un texte en langue de départ et sa traduction en langue d'arrivée à la recherche de stratégies utilisées dans ce processus, la présente étude table sur les énoncés de la langue d'arrivée pour les retracer dans la langue de départ afin de détecter les déviations intervenues dans cette dernière. En effet, les énoncés du cilubà sont plus élaborés que leurs sources latines et françaises comme conséquence de leur inculturation dans ces dernière langues.

Concernant l'ethnographie de communication, nous nous sommes intéressé aux stratégies langagières utilisées par les officiants, les modérateurs des messes et les chorales pour transmettre d'une façon adéquate le message liturgique d'origine juive aux croyants lubà. Notre approche est purement descriptive en ce qu'elle consiste à expliciter ces messages imagés en rapport avec les textes latins et français d'où ils tirent leur origine pour prouver leur indigénisation.

\section{Quelques considérations sur la traduction}

La traduction est une vieille discipline dont la longue tradition remonte aux anciennes civilisations, notamment à la culture grecque, si pas plus loin encore dans le temps. Plusieurs définitions de la traduction sont en vogue. Par exemple, pour Taber et Nida (1971: 11), «la traduction consiste à reproduire dans la langue réceptrice le message de la langue source au moyen de l'équivalent le plus proche et le plus naturel, d'abord en ce qui concerne le sens et ensuite en ce qui concerne le style». Bien plus, pour théoriser explicitement sur l'objectif de communication de traduction en fonction de récepteurs précis Nida (1964) a défini deux concepts d'équivalence entre le texte de départ et le texte d'arrivée: l'équivalence formelle et l'équivalence dynamique. La première cherche à reproduire la forme du texte de départ tandis que la dernière cherche à répondre aux besoins du destinataire.

$\mathrm{Au}$ niveau théorique, deux paradigmes semblent se dégager dans les différentes écoles de la traduction. Nonobstant les différences terminologiques, les paradigmes se ramènent à la dichotomie entre «la traduction sémantique» et «la traduction fonctionnelle». En fait, selon Kambaja (2007: 50) «la traduction est dite sémantique lorsque elle tente, selon les contraintes syntaxiques et sémantiques de la langue d'arrivée, de reproduire la signification contextuelle précise du texte de départ». Par contre, la traduction fonctionnelle est celle qui vise les équivalences dans l'accomplissement de la même fonction au-delà de différences de formes. Dans le même ordre d'idées, Gonzalez (2003) déclare:

L'équivalence fonctionnelle consiste à atteindre, au moyen des éléments linguistiques, extralinguistiques (ou contextuels) et culturels, le même but, la même fonction que le texte source. Il faudrait donc que le texte cible produise les mêmes actes pragmatiques que le texte source. 
Il identifie les types d'équivalences suivantes: l'équivalence linguistique, l'équivalence pragmatique, l'équivalence stylistique, l'équivalence sémantique, l'équivalence formelle, l'équivalence référentielle, l'équivalence paradigmatique, l'équivalence dynamique et l'équivalence fonctionnelle.

À côté de cette terminologie technique existe celle courante qui classifie la traduction en «traduction littérale» et «traduction littéraire ou culturelle» qui correspondent respectivement à la «traduction sémantique» et «la traduction fonctionnelle» susmentionnées. En définitive, le débat tourne autour de la fidélité à observer par rapport au texte de départ ou de la langue source.

Il sied cependant de noter que la traduction est avant tout traduction du discours et non traduction des mots pour que l'on puisse s'en tenir aux équivalences sémantiques. La notion d'équivalence implique la comparaison et l'égalité des entités en présence. Les récentes tendances de la traductologie dont la théorie interprétative (Seleskovitch et Lederer 2001) et la théorie de pertinence (Sperber et Wilson 1986) illustrent bien ce débat sur l'objet réel de la traduction. Elles reconnaissent que l'on traduit non pas les langues en présence, mais le sens que l'on a compris comme celui que le contexte confère à l'énoncé. Luscher (2001) parle de la traductologie du discours qui considère l'énoncé comme unité de traduction.

Comme nous le verrons dans l'interprétation des données, l'inculturation du message liturgique amène le traducteur et l'interprète à restituer le discours et non les simples structures grammaticales ou lexicales. Nous pouvons conclure ce débat avec Kambaja (2007: 53) qui stipule ceci: «En tant qu'acte de communication, la traduction ne peut être étudiée que sous un angle pragmatique, prenant en compte les aspects sémantiques, cognitifs, logiques et encyclopédiques.» Salah et al. (2003) résument mieux ces préoccupations par le titre de leur livre Traduire la langue, traduire la culture. Tous ces facteurs sont sous-entendus dans «le contexte» qui définit ainsi la culture.

Sur le plan pratique, la traduction nécessite des stratégies variées pour rendre le message de la langue de départ à la langue d'arrivée le plus fidèlement possible en dépit de l'aphorisme italien «traduttore, traditore», c'est-àdire, traduire c'est trahir. Selon Eno Belinga (1978: 58), cet aphorisme «établit fatalement que toute traduction est infidèle puisqu'elle trahit la pensée du texte original». Parmi les stratégies le plus en vue, nous pouvons mentionner le chassé-croisé, l'explication, le remplacement métaphorique, l'équivalence dynamique, l'ajout métaphorique, la traduction littérale etc.

La traduction exige du traducteur un effort de créativité dans le choix des termes propres et expressifs et des structures syntaxiques. La qualité de la traduction dépend ainsi du génie créateur du traducteur à manier la langue d'arrivée tant dans sa forme orale qu'écrite.

Comme soubassement théorique, dans les lignes qui suivent, nous explorons certaines sources des difficultés réelles inhérentes à la traduction du langage imagé. Nous essayons d'illustrer comment la même réalité est rendue dans ces trois langues: le français, l'anglais et le cilubà. 
Certains énoncés sont difficiles à traduire parce que les langues en présence perçoivent les réalités différemment. Le traducteur doit procéder à la traduction fonctionnelle pour rendre le message compréhensible dans la langue cible même si cela doit entraîner des contradictions apparentes. En linguistique contrastive, tel est souvent le cas en lexicologie pour les termes considérés comme ayant des «sens étranges». Par exemple, l'heure n'est pas comptée de la même façon en kiswahili et dans certaines langues européennes. Ainsi, 9 heures $d u$ matin est «nine o'clock» en anglais mais «saa tatu» en kiswahili, alors que tatu signifie «trois» et non «neuf». La raison reste simple: les occidentaux comptent les heures du jour après minuit. Par contre, les waswahili commencent leur journée au lever du soleil. Ainsi se retrouvent-ils en retard de six heures par rapport aux occidentaux.

D'autres exemples peuvent aussi illustrer ce phénomène:

(1)(a) Filer à l'anglaise

se traduit en anglais comme

(b) To take French leave

mais en cilubà, respectivement comme

(c) Kuongoloka (quitter en catimini)

et

(d) Kuongoloka bu kabundi (quitter en catimini comme une genette)

French n'est pas la traduction anglaise de l'adjectif anglaise. Il est curieux de constater que les Français et les Anglais s'accusent mutuellement sur la façon de quitter un lieu en catimini. Pour exprimer la même réalité, le peuple lubà recourt à deux énoncés dont l'un est une comparaison avec la genette, un animal réputé rusé dans les fables pour son intelligence.

Dans les proverbes et autres formes de langage figé, là où telle langue utilise comme symbole un animal, telle autre peut utiliser un oiseau, un animal différent, etc.

(2)(a) Il ne faut pas vendre la peau de l'ours avant de l'avoir abattu/tué

(b) Don't count your chickens before they are hatched

(c) Nkàsù bàtu bàyìmwèna mu milabì (Les houes, on les voit dans les manches)

La certitude, ou mieux, la méfiance sur les résultats futurs est rendue en français au moyen de l'animal «ours» (bear), en anglais par «chickens» (poules) et en cilubà par «nkàsù» (houes). Pour réussir dans cette entreprise, le traducteur doit recourir à l'équivalence fonctionnelle pour ne pas trahir le message de la langue source. 
Bien plus, au niveau de structures des langues, on peut passer de telle partie du discours à telle autre, avec souvent l'élaboration ou la condensation du message:

(3)(a) Mieux vaut prévenir que guérir

(b) Prevention is better than cure

(c) Budìmù m̀bupite bwanga (La prudence dépasse le fétiche)

Le français utilise les verbes (prévenir et guérir) là où l'anglais utilise les noms (prevention et cure). Le cilubà recourt aussi aux noms mais donne l'équivalent et non la traduction proprement dite de l'aphorisme français. Le français et l'anglais opèrent au sein du même champs sémantique (prévenir, prevention) tandis que le cilubà utilise les termes d'un autre domaine (budìmù et bwanga).

Certaines traductions sont basées sur la recherche des mêmes effets dans les deux langues:

(4)(a) Monsieur Dupond et Monsieur Dupont

(b) Mr Thompson and Mr Thomson

Il s'agit de personnages des bandes dessinées Tintin. Pour produire l'ambiguiité créée par les homophones, le traducteur de l'énoncé français en anglais a fait recours aux noms anglais qui sont aussi épelés différemment mais sont prononcés de la même façon.

Enfin, la traduction des énoncés de langues africaines en langues européennes exige souvent le recours à «la composition» comme procédé linguistique. Celle-ci permet au traducteur de combiner les éléments de deux langues pour exprimer avec précision une idée qui resterait vague si le terme générique était employé. Ainsi, ces devises sont traduites comme suit:

(5)(a) Dijinda ntùnga mùlòngò

(b) La fourmi-dijinda (la tête de file des fourmis)

(c) The ant-dijinda (the leader of the line of ants)

Il y a plusieurs espèces de fourmis en cilubà, chacune ayant ses propres habitudes et son propre comportement. Traduire dijinda simplement comme fourmi créerait de la confusion, ou pire, manquerait de mettre en lumière la qualité de «meneur» ou de "guide» spécifique à cette espèce, qualité que l'on aimerait attribuer métaphoriquement au référent (Jésus Christ pour le cas d'espèce).

(6)(a) Cinkùnkù nsanga bilembi

(b) L'arbre-cinkùnkù (rassembleur des chasseurs)

(c) Cinkùnkù-tree (gatherer of the hunters)

Comme pour le cas précédent, la composition est très importante pour maintenir le terme cinkùnkù dans le lexème. Cela permettrait de conserver le sens profond de cette devise, à savoir, la qualité de rassembleur reconnu au référent. 
En guise de conclusion, il revient donc au traducteur et à l'interprète de choisir les stratégies de traduction appropriées pour rendre le message plus fidèlement dans la langue d'arrivée.

\section{Ethnographie de communication}

Un autre aspect visible de l'indigénisation du rite religieux reste sans doute l'adoption de l'ethnographie de communication des cultures africaines. Pour toucher les croyants dans leur moi-profond, le modérateur, l'officiant et même le compositeur des cantiques essayent de rapprocher le message liturgique de la philosophie et culture de ceux-ci. D'où l'emploi du langage imagé, notamment les aphorismes, c'est-à-dire, les proverbes au sens large.

En effet, les proverbes jouent un grand rôle dans le discours africain. Ils sont les signes visibles de l'expression de la sagesse non seulement du sujet parlant, mais aussi de celle de l'auditoire pour lequel le langage est adapté en conséquence. Selon Schmied (1991: 52):

L'une de fonctions du proverbe est de résumer les points qui peuvent être difficiles à expliquer. Bien plus, les proverbes montrent la sagesse du locuteur ... Ils neutralisent ce qui serait autrement des vérités désagréables ou dangereuses pour l'auditeur.

Dans le même ordre d'idées, Oladele (1981: 26) écrit:

Les proverbes concernent tous les aspects de la vie. Ils sont utilisés pour exprimer avec force les paroles des sages et font partie du patrimoine de vieilles personnes, qui les utilisent pour transmettre des leçons morales précises, avertissements et conseils parce qu'ils font un grand impact sur l'esprit plus que les mots ordinaires.

Lors de cérémonies religieuses, les proverbes sont souvent utilisés par le modérateur pour introduire les lectures saintes, mais presque toujours pour les conclure ou les résumer.

Par ailleurs, sur le plan d'interaction, nous assistons à un dialogue entre le modérateur et le public. Le premier pose une question ou prononce une formule introductive de manière à préparer le public sur le sens des lectures saintes ou du message à venir. Comme conséquence, un même texte liturgique peut être introduit ou conclu au moyen de parémies différentes selon la créativité et l'inculturation du modérateur ou de l'officiant, ou selon l'exégèse que chacun d'eux fait de la lecture elle-même.

Mais que représente tout ceci pour le traducteur, se demanderait-on? En fait, bien que le modérateur et l'officiant utilisent un langage simple et ordinaire pour mieux communiquer avec le public, le souci de sonner sage les pousse souvent à employer les parémies. Celles-ci prennent souvent l'interprète au dépourvu car elles exigent une interprétation rapide du message afin 
de procéder à la traduction fonctionnelle, à la traduction libre ou à l'explication de l'acte langagier comme en cas d'onomatopées.

Parfois certains animateurs et modérateurs utilisent des parémies n'ayant aucune relation directe avec le message liturgique. C'est-à-dire, le contexte n'est pas approprié pour faciliter la traduction à cause de la discordance entre l'acte posé et le message utilisé pour l'annoncer. Ce qui complique la tâche de l'interprète. À titre illustratif, pour annoncer le port des étoles par les nouveaux prêtres comme symbole de leur nouvelle identité, le prêtre modérateur du jour utilisera cette parémie:

Modérateur:

(7)(a) Bulenga bwà cibangà

(b) La beauté du menton

(c) The beauty of the chin

Public:

(8)(a) m̀mmevu

(b) c'est la barbe

(c) is the beard

Non seulement le contexte de cet usage n'est pas approprié, mais la véracité même de cette parémie reste relative car le lien entre la barbe et la beauté masculine n'est pas une valeur universelle.

En résumé, grâce au renouveau liturgique, le modérateur, l'officiant et le public ne sont plus relégués au simple rôle des répétiteurs des paroles figées latines dont ils ne comprenaient pas toujours le sens comme dans le cas des formules incantatoires ou magiques. Ils sont devenus créateurs des discours qui utilisent le message liturgique comme simple point de départ et puisent dans la cosmologie et la philosophie lubà dans leur conception des actes langagiers.

\section{Essai de traduction des énoncés religieux}

Dans cette entreprise, nous fournissons chaque fois un énoncé en cilubà, suivi de sa traduction en français et en anglais. Ensuite, nous ajoutons d'autres énoncés qui attestent l'indigénisation du message et présentent de sérieuses difficultés de traduction pour l'interprète et le traducteur. Certes, l'emphase sera placée sur le message en cilubà pour évaluer comment les traducteurs ont atteint l'africanisation du message liturgique.

(9)(a) Mwâna wa balùmè umwe

(b) Le fils unique

(c) The only Son

(d) mwâna mulela umwe: un enfant né seul 
(e) mulela umwe: né seul

(f) Nkònga mikùjà wa Màriyà: rassembleur des fétus de Marie

Pour le cilubà nous enregistrons plusieurs traductions selon les degrés d'inculturation. Comme on peut le remarquer, l'équivalence paradigmatique semble parfaite entre les expressions françaises et anglaises. Nous avons donc deux lexèmes, si nous considérons l'article en anglais et en français comme un élément grammatical sans contenu sémantique.

En cilubà, nous avons l'expression mwâna wa balùmè umwe. Sa traduction littérale signifie «un garçon» et non pas «le fils unique». D'où l'emploi de l'expression appropriée mwâna mulela umwe «un enfant né seul» trouvée dans le Credo par exemple. Celle-ci est même souvent réduite à la devise mulela umwe «né seul» (Kabasélé 1986a: 264) dans laquelle le terme mwâna «enfant» est omis ou reste sous-entendu.

Enfin, le cantique utilise la devise nkònga mikùjà wa Màriyà, un langage imagé qui témoigne d'une bonne connaissance de la culture lubà par le compositeur. Cette expression signifie littéralement «le fétus qui rassemble tous les fétus de Marie» et connote que cet enfant spécial rassemble en son sein les attributs de tous les enfants potentiels de Marie. Ce qui est une image de richesse et non celle de stérilité ou de pauvreté de fécondité inhérente à la notion de fils unique chez l'africain.

(10)(a) Kubèènesha

(b) Bénir

(c) To bless

(d) kwela lupèmbà

(e) kutentekeela byanza

Kubèènesha est un emprunt linguistique français assimilé dans lequel on identifie facilement la racine ben- provenant de bénir. A part cet emprunt, nous avons aussi kwela lupèmbà «asperger avec du caolin», une expression native qui trouve son origine dans les pratiques animistes propres à la plupart de sociétés africaines.

Kubèènesha et kwela lupèmbà diffèrent non seulement par leurs origines respectives, mais aussi par la paralinguistique qui les accompagne. En tant que symbole du christianisme, kubèènesha est associé avec le signe de la croix alors que kwela lupèmbà sous-entend l'aspersion au moyen du caolin ou le dessin au caolin sur le front et les joues du sujet.

Enfin, dans les confessions religieuses où le signe de croix n'est pas de mise, on pratique l'imposition des mains (kutentekeela byanza). Néanmoins, même au sein de l'Église Catholique Romaine, cette pratique est courante dans le Mouvement Charismatique et autres dont le rite religieux se rapproche de plus en plus de ceux des Églises de Réveil.

(11)(a) Kutooka zeezeeze 
(b) Être pur

(c) To be pure/cleansed

Des termes natifs et des emprunts linguistiques existent côte à côte pour des raisons souvent peu évidentes. De plus, la dualité des termes est entretenue parfois même là où l'emprunt linguistique pouvait être évité comme dans ces extraits des cantiques:

(d) ... ǹshaalà zeezeeze_Mfùmù wanyì

... que je sois très pur, Mon Seigneur

... provided that I remain pure, my Lord

(e) .... ǹtookà mupìta nêjè

... que je devienne plus blanc que la neige

... provided that I become whiter than snow

(f) ... mutòòke bu bùùsù bwà ndânda

... aussi blanc (pur) qu'un flocon de coton

... as white (pure) as a flock of cotton

Dans ces extraits, la notion de pureté est exprimée en terme de «blancheur». Dans le premier cantique (11)(d), nous avons un terme natif (kuooka) zeezeeze (très blanc). Dans le deuxième (11)(e), nous avons l'emprunt linguistique français nêjè (neige). Enfin, dans le troisième (11)(f), nous avons l'image locale bùùsù bwà ndânda (flocon de coton). Sa traduction littérale est «flocon de coton», tandis que sa traduction fonctionnelle doit être «flocon de neige», la mesure de blancheur dans la langue française étant attestée par l'expression blanc comme neige.

Les parémies présentent plus de difficultés de traduction que les lexèmes simples. Ainsi, là où le dialogue est engagé, l'interprète doit souvent attendre la fin de l'échange dans son contexte afin de concevoir sa traduction:

Modérateur:

(12)(a) Bààya

(b) S'ils partent

(c) If they go

Public:

(13)(a) Wâya

(b) Pars (aussi)

(c) Go (also)

Modérateur:

(14)(a) Bààshààla

(b) S'ils restent

(c) If they stay 
Public:

(15)(a) Washààdilamù

(b) Reste pour toujours

(c) Stay for ever

La traduction littérale ci-dessus n'a pas de sens. En fait, il s'agit ici d'une simple interaction de nature à attirer l'attention du public sur le message important à venir. À la fin de cet échange, l'interprète peut alors exploiter le contexte et procéder à la traduction fonctionnelle au moyen de parémies suivantes pour permettre aux étrangers de saisir le message:

(d) Il faut hurler avec les loups

(e) When in Rome, do as the Romans do.

Toutes ces traductions invitent l'assistance à saisire les couleurs locales, c'est-àdire, à s'adapter au contexte ou à suivre l'exemple des autres membres de la communauté que l'on rejoint.

D'autres parémies, bien que traduisibles, nécessitent des explications supplémentaires pour rendre le message explicite:

Modérateur:

(16)(a) Cimanyinu cyà bânà bàà Kabòngù

(b) Le signe distinctif des enfants de Kabòngù

(c) The distinctive sign of Mr Kabòngù's children

Public:

(17)(a) ǹkabùkì mu nshìngù

(b) c'est le pendentif au cou

(c) is an amulet on the neck

Cette parémie a été utilisée à diverses occasions pour annoncer le port des étoles par les nouveaux prêtres et diacres, des chapelets par les nouveaux baptisés et premiers communiants et des croisettes par les jeunes de lumière promus. Les modérateurs voulaient chaque fois attirer l'attention du public sur l'acte que l'officiant allait exécuter bientôt pour conférer une nouvelle identité aux lauréats.

Pour faire participer les étrangers à ces actes langagiers, l'interprète peut tout au plus leur expliquer la cérémonie en ces termes:

(18)(a) Maintenant l'officiant va procéder à ...

(b) Now the priest is going to ...

Certaines parémies, quoique traduisibles littéralement, requièrent une traduction fonctionnelle pour révéler la sagesse dans la langue cible: 
Modérateur:

(19)(a) Nzùbù mukòsòlòka ...

(b) Une maison divisée ....

(c) A house divided against itself ...

Public:

(20)(a) Cyèndà ǹkabùtù

(b) La chute est son sort

(c) To fall is its fate

Ces traductions peuvent être améliorées au moyen de parémies françaises et anglaises comme suit:

(d) L'union fait la force

(e) United we stand, divided we fall.

Une certaine catégorie de parémies ne se prête à aucune traduction. Il s'agit particulièrement des onomatopées. L'interprète peut tout au plus en expliquer le sens ou l'usage:

Modérateur:

(21) Petèètèpêtè èè ...

(Onomatopée)

(Onomatopoeia)

Public:

(22)(a) Patwàkashìla dîyì

(b) Là où nous avons laissé la voix

(c) There where we left the voice

La traduction fonctionnelle en français et anglais est respectivement:

(d) Notre convention était ...

(e) Our agreement was...

Le modérateur utilise une onomatopée tandis que le public répond littéralement «là où nous avons laissé notre convention», c'est-à-dire, «notre convention était ...». L'interprète peut expliquer à ses hôtes cet acte en ces termes:

(23)(a) Il leur rappelle la convention concernant ...

(b) $\mathrm{He} / \mathrm{She}$ reminds them of the agreement about ...

Dans leur traitement de la notion d'équivalence, Vinay et Darbelnet (1958: 52) ont abordé le cas des onomatopées en spécifiant que «les deux textes rendent compte d'une même situation en mettant en œuvre des moyens stylistiques et structuraux entièrement différents». Ainsi illustrent-ils le cri de douleur par 
Aie! en français et Ouch! en anglais, auxquels nous pouvons ajouter Eyi/Ayi! en cilubà. Petèètèpêtè èè est une formule introductive d'une interaction qui n'a pas d'équivalent dans l'ethnographie de communication du français et de l'anglais.

Enfin, la cosmologie lubà nous mène parfois dangereusement à la limite entre le christianisme et le paganisme; ce qui rend la traduction bizarre. Par exemple, traduire la devise

(24)(a) Mwanza-nkòngòlò lukànda mvùla wa mudimbì

comme

(b) L'arc-en-ciel qui arrête la pluie torrentielle

(c) The rainbow which stops the torrential rain

serait ériger un simple élément cosmique en une divinité ou force cosmique guerrière. Et pourtant la Bible reconnaît l'arc-en-ciel tout simplement comme le signe de l'alliance de paix entre le Seigneur et Noé après le Déluge (Genèse 9: 13). Ainsi, les devises de Jésus, de Dieu ou de la divinité en général sont tirées de divers domaines et associées avec toutes les forces de la nature dont les arbres, les animaux, les oiseaux, les êtres mythiques et mythologiques, les phénomènes cosmiques et atmosphériques etc. (voir Kabasélé 1986a, 1986b).

L'accumulation des devises est fréquente dans les cantiques où les compositeurs louent Jésus Christ en invoquant ses divers attributs ensemble. Cette strophe d'une version de Gloria en est bien un exemple significatif:

(25)(a) Mukalenga wa diulu ...

(b) Le Seigneur du Ciel

(c) The Heavenly King

(26)(a) Mwena ngulu yônso ...

(b) Le Tout-Puissant

(c) The Almighty

(27)(a) Ntìita lwàbanya màkàlèngà ...

(b) Ntìta, le donneur du pouvoir

(c) Ntìta, the giver of power

(28)(a) Ngabu wetù wa kweyemena

(b) Notre bouclier sur lequel s'appuyer

(c) Our shield on which to lean

(29)(a) Mwâna mukòkò ...

(b) L'agneau de Dieu ...

(c) The Lamb of God ...

(30)(a) Mâyi a kowa ... àdì àtòòkesha bantu

(b) L'eau à laver ... qui purifie les gens 
(c) The water used for washing ... which cleanses the people

(31)(a) Mulopò cyenda ne bantu

(b) Dieu qui chemine avec le peuple (Dieu, le guide du peuple)

(c) God who walks with his people (God, the people's guide)

Toutes ces devises se traduisent simplement par le référent «Jésus Christ», et sont choisies selon le contexte et l'attribut que l'on aimerait mettre en lumière.

En bref, traduire un énoncé liturgique du cilubà en langues européennes requiert moins la connaissance de l'équivalent de chaque mot que celle de la correspondance de la réalité entre les langues et les cultures, de que Taber et Nida (1971) ont qualifié de «traduction pragmatiquement équivalente». En effet, une tâche difficile pour l'interprète du cilubà qui ne dispose pas d'assez de temps pour rendre fidèlement l'énoncé dans la langue cible.

\section{Conclusion}

Notre objectif dans cette étude consistait à mettre en évidence les difficultés de traduire les énoncés religieux du cilubà en français et en anglais. Ensuite, notre recherche a egalement voulu démontrer qu'en libéralisant l'utilisation des langues locales dans le rite religieux, le renouveau liturgique catholique a aussi libéré le croyant lui-même dans le domaine langagier. Plutôt que de rester lié à la structure et au contenu du message liturgique tel que le traduisent les écritures saintes, le croyant mulubà en l'occurrence, utilise, par contre, ces messages comme source d'inspiration pour exprimer sa foi selon sa propre culture. Tel est surtout le cas pour les cantiques et les discours des officiants et des modérateurs des messes qui sont parsemés de devises et de langage imagés. Le croyant intègre à la Bonne Nouvelle la cosmologie de sa culture et l'ethnographie de communication de sa propre langue. Dans cet effort d'indigéniser le message liturgique, le traducteur et l'interprète sont obligés d'utiliser la traduction fonctionnelle afin de respecter et de sauvegarder les normes communicationnelles de la langue d'arrivée qu'est le cilubà. Par conséquent, le message devient tellement inculturé qu'il est difficile de le restituer encore fidèlement aux locuteurs des langues européennes à cause des africanismes qu'il contient désormais.

Sur le plan de l'ethnographie de communication, le croyant africain peut bien accueillir le message liturgique apporté par l'européen, sans pour autant renoncer à sa propre culture. Tout comme les juifs déportés à Babylone qui demandaient à leurs détracteurs «Comment chanterions-nous les cantiques de l'Éternel sur une terre étrangère?» (Ps 137: 4), les croyants lubà du renouveau liturgique catholique doivent s'être également demandé, mutatis mutandis, «Comment louerions-nous notre Créateur Mawêja-Nààngila, notre Mikombe-aKalèèwu dans un langage étranger?» D'où le recours au langage imagé parsemé des devises et des proverbes, et à la paralinguistique propre au cilubà. 
Rendre toute cette richesse culturelle en langues européennes susmentionnées est une tâche ardue pour l'interprète et le traducteur.

\section{Bibliographie}

Eno Belinga, Samuel-Martin. 1978. Comprendre la littérature orale africaine. Yaoundé: Éditions St Paul.

Faïk-Nzuji, Clementine M. 1974. Kàsàlà, chant hérö̈que lubà. Lubumbashi: Presses universitaires du Zaïre.

Gile, Daniel. 2007. La traduction. La comprendre, l'apprendre. Paris: Presses universitaires de France.

Gonzalez, Gladys. 2003. L'équivalence en traduction juridique: Analyse des traductions au sein de l'Accord de libre-échange Nord-Américain (ALENA). Thèse de doctorat. Québec: Université Laval, Département des langues, linguistique et traduction. http://www.theses.ulaval.ca/2003/ 21362/21362.html [26 March 2008].

Guthrie, Malcolm. 1948. The Classification of Bantu Languages. Oxford: Oxford University Press.

Kabasélé, François. 1986a. Christ dans l'actualité de nos communautés. Luneau, René, Joseph Doré et François Kabasélé (Éds.). 1986. Chemins de la christologie africaine: 263-272. Paris: Desclée.

Kabasélé, François. 1986b. Le Christ comme chef. Luneau, René, Joseph Doré et François Kabasélé (Éds.). 1986. Chemins de la christologie africaine: 109-125. Paris: Desclée.

Kachru, Braj B. 1982. The Other Tongue: English across Cultures. Urbana, Ill.: University of Illinois Press.

Kambaja, M. 2007. Approche pragmatique de la traduction français-cilubà. Contribution à la théorie traductologique. Mémoire de D.E.S. Lubumbashi: Université de Lubumbashi, Département des sciences du langage.

Luscher, Laure A. 2001. Vers une théorie sémantico-pragmatique pour la traduction. Göppigen: Kümmerle Verlag.

Newmark, Peter. 1982. Approaches to Translation. Oxford: Pergamon.

Nida, Eugene A. 1964. Toward a Science of Translating with Special Reference to Principles and Procedures Involved in Bible Translating. Leyde: E. J. Brill.

Oladele, Taiwo. 1981. An Introduction to West-African Literature. Nairobi: Nelson.

Salah, Mejri, Bacchouche Taieb et André Class (Éds.). 2003. Traduire la langue, traduire la culture: rencontres linguistiques méditerranéennes. Paris: Maisonneuve et Lerose.

Schmied, Joseph. 1991. English in Africa: An Introduction. Londres: Longman.

Seleskovitch, Danica et Marianne Lederer. 2001. Interpréter pour traduire. 4ème édition. Paris: Didier Érudition.

Sperber, Dan et Deirdre Wilson. 1986. La pertinence: communication et cognition. Paris: Édition de Minuit.

Taber, Charles et Eugene A. Nida. 1971. La traduction: théorie et méthode. Londres: Alliance Biblique Universelle.

Vinay, Jean-Paul et Jean Louis Darbelnet. 1958. Stylistique comparée du français et de l'anglais: méthode de traduction. Paris: Didier. 